\title{
XMM-Newton X-ray spectra of the SNR 0509-67.5: data and models
}

\author{
D. Kosenko ${ }^{1,3}$, J. Vink ${ }^{1,2}$, S. Blinnikov ${ }^{4,3}$, and A. Rasmussen ${ }^{5}$ \\ 1 Astronomical Institute Utrecht, University Utrecht, PO Box 80000, 3508TA Utrecht, The Netherlands \\ e-mail: D.Kosenko@astro.uu.nl \\ 2 SRON, Utrecht, The Netherlands \\ 3 Sternberg Astronomical Institute, Russia \\ 4 Institute for Theoretical and Experimental Physics, 117218 Moscow, Russia \\ 5 Stanford Linear Accelerator Center, Menlo Park, CA, USA
}

Received 1 February 2008 / Accepted 26 June 2008

\section{ABSTRACT}

\begin{abstract}
Aims. We report on X-ray observations of the supernova remnant 0509-67.5 in the Large Magellanic Cloud acquired by the XMMNewton X-ray observatory. We use the imaging spectroscopy (EPIC) and Reflective Grating Spectrometer (RGS) data to investigate properties of the remnant and its environment.

Methods. The X-ray spectra were analyzed with SPEX software package. In addition, we performed a numerical hydrodynamic simulation of the remnant.

Results. The EPIC data show prominent Fe $\mathrm{K}$ line emission, but the deduced overall amount of iron in the shocked ejecta is low. The data also show that the remnant has an asymmetric ejecta structure: the bright southwest region of the remnant shows an overabundance of metals. The analysis of the RGS spectrum shows that the remnant has a high line velocity broadening of $5000 \mathrm{~km} \mathrm{~s}^{-1}$. We developed a hydrodynamical model for the remnant with basic hydrodynamical and spectral parameters that are similar to those observed.

Conclusions. The data analysis indicates that the reverse shock just recently reached iron layers of the ejecta. The brightness enhancement in the southwest region could be a sign of an asymmetric explosion or it could be the result of a density enhancement in the interstellar medium. We constructed numerical models that are in good agreement with the observations, with circumstellar density of $3 \times 10^{-25} \mathrm{~g} / \mathrm{cm}^{3}$, age of $\sim 400$ years, velocities of $\sim 5000 \mathrm{~km} \mathrm{~s}^{-1}$, and an electron-to-ion temperature ratio of $10^{-2}$.
\end{abstract}

Key words. X-rays: individuals: SNR 0509-67.5 - ISM: individual objects: SNR 0509-67.5 - ISM: supernova remnants methods: data analysis - hydrodynamics

\section{Introduction}

The X-ray emission from young supernova remnants (SNRs) provide a vital source of information about the properties of both shocked, rarefied plasma and the ambient medium around the remnants, and supernova explosion models. With the current generation of X-ray telescopes, XMM-Newton, Chandra, and Suzaku, we are now able to combine the imaging and spectral information to study the extended emission from SNRs. In the case of Chandra and XMM-Newton, dispersive spectrometers allow high resolution spectra to be obtained, providing more constraints on both the plasma parameters and the dynamics of the plasma, by means of Doppler shifts and broadening. The XMMNewton Reflection Grating Spectrometers (RGS) have the advantage that the spectral quality is less deteriorated by the spatial extent of the object, at least for sources with an angular extent of $\lesssim 1^{\prime}$

SNRs in the Large Magellanic Cloud (LMC) and the Small Magellanic Cloud (SMC) are particularly able targets, since the relative proximity of the LMC means that many SNRs are sufficiently bright for high signal-to-noise spectra to be obtained with both the CCD and grating spectrometers. On the other hand, the distance to the LMC, $50 \mathrm{kpc}$, is such that young SNRs have an extent of less than $1^{\prime}$, making them excellent targets for the XMM-Newton RGS. The XMM-Newton CCDs, with a resolution of $\sim 6^{\prime \prime}$, still allows one to study spatial variations, although Chandra obviously provides more spatial details. In addition, studying SMC/LMC remnants has the advantage that the interstellar absorption is on average lower than for most of the Galactic remnants, and their distance is known.

XMM-Newton observations of several LMC/SMC remnants have been published: N132D (Behar et al. 2001), 1E 0102.27219 (Rasmussen et al. 2001; Sasaki et al. 2006), B0540-69.3 (van der Heyden et al. 2001), N103B (van der Heyden et al. 2002), and several SMC remnants (van der Heyden et al. 2004).

Here we report on the X-ray spectrum of the SNR 0509-67.5 in the Large Magellanic Cloud. This object was already examined by several authors, e.g. Warren \& Hughes (2004), Vink (2006), Badenes et al. (2008), Rest et al. (2008), Ghavamian et al. (2007), Rest et al. (2005), Badenes et al. (2007), and Hendrick \& Reynolds (2001). The remnant has a spherical shape and is similar in age and size to Tycho's SNR, which is also a Type Ia SNR. SNR 0509-67.5 has an angular diameter of 25", corresponding to a radius of 3.6 pc (Warren \& Hughes 2004). Analyses by both Rest et al. (2005), based on light echo detection, and Badenes et al. (2008), based on modeling of the X-ray observation, suggested that the age of the remnant was $\sim 400$ years. Estimates by Ghavamian et al. (2007) provide the values of 295-585 years, which are consistent with the rough estimate $\sim 500$ years by Vink (2006), based on a preliminary analysis of the XMM-Newton data.

A detailed analysis of the X-ray emission of SNR 0509-67.5 was completed by Warren \& Hughes (2004) based on Chandra 

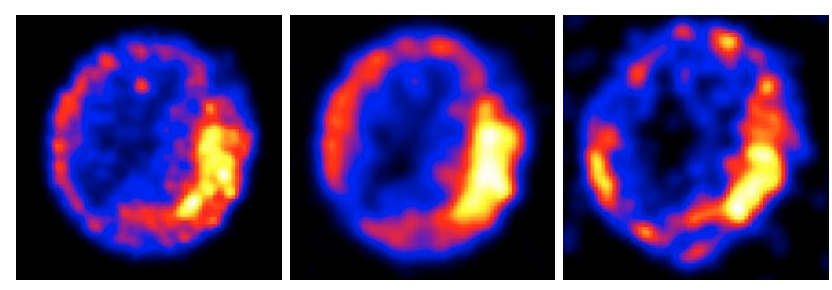

Fig. 1. Chandra images of the SNR 0509-67.5 in different X-ray ranges. From left to right: $0.45-1.75 \mathrm{keV}, 1.8-2.5 \mathrm{keV}, 2.5-6.0 \mathrm{keV}$ (Warren \& Hughes 2004).

X-ray data. Their study revealed that the remnant is rich in silicon, sulfur, and iron, that the bulk of the continuum emission has a non-thermal origin, and that the circumstellar medium (CSM) density is approximately $0.05 \mathrm{~cm}^{-3}$. The preferred model for the explosion that produced the remnant is delayed detonation.

Badenes et al. (2008) studied the X-ray spectrum of the remnant obtained from Chandra and XMM-Newton observations. They performed hydrodynamical and X-ray spectral calculations of the remnant and concluded that it was an energetic, delayed detonation explosion of $1.4 \times 10^{51}$ erg with a nickel mass of $0.97 M_{\odot}$. In their numerical simulations, the circumstellar density was set to $n_{\mathrm{CSM}}=0.4 \mathrm{~cm}^{-3}$ and the ratio of electron-to-ion temperatures to 0.02 .

For illustrative purposes in Fig. 1 we show some Chandra $\mathrm{X}$-ray images of the remnant in three different bands. The overall structure of the SNR shows a large-scale inhomogeneity in the shell. The southwest (SW) part of the remnant is brighter compared to the average brightness.

In the current study, we performed an analysis of the remnant employing XMM-Newton X-ray observations and SPEX fitting software (Kaastra et al. 1996). We also performed hydrodynamical simulations of the SNR 0509-67.5, employing the hydrocode SUPREMNA (Sorokina et al. 2004) to compare numerical predictions for the remnant with observations.

The paper is organized as follows. First, we describe briefly the XMM-Newton data in Sect. 2, then we discuss the X-ray spectrum in Sect. 3. Numerical models for the SNR are presented in Sect. 4. We discuss our results and outline them in Sect. 5.

\section{The $X M M-N e w t o n$ data, reduction and analysis}

SNR 0509-67.5 was observed with the XMM-Newton X-ray Observatory (obs ID 0111130201 ) for $35.9 \mathrm{ks}$ on July 4, 2000. The observatory contains three X-ray telescopes. Behind all of them are CCD detectors, collectively called the European Photon Imaging Camera (EPIC). Two of them are MOS type CCD detectors called MOS1 and MOS2 (Turner et al. 2001), and the other is of pn-CCD type (Strüder et al. 2001). The telescopes equipped with MOS CCD detectors in the focal plane contain also the Reflective Grating Spectrometers (RGS), consisting of the Reflective Grating Arrays, which disperse about 50\% of the $\mathrm{X}$-rays into two CCD arrays (den Herder et al. 2001). XMMNewton observations therefore provide simultaneous data from all five X-ray instruments: the two MOS-CCDs, the pn-CCD, and the two RGS instruments.

In this paper, we concentrate on the data obtained with the MOS detectors and the RGS. Although the EPIC-MOS have a lower sensitivity than the EPIC-pn instrument, they have higher spectral resolution, which is important for line rich sources, such as SNRs.

We note that Badenes et al. (2008) found an inconsistency between EPIC MOS1,2 and PN spectra. They investigated and compared the $\mathrm{Si} \mathrm{K}_{\alpha}$ line centroid location in both data sets, and found that the line centroids of the MOS and PN detectors are shifted with respect to each other. The authors disregarded the MOS1 and MOS2 data and considered only the Chandra and EPIC-pn spectra. We confirm this inconsistency: analysis of the Si $\mathrm{K}_{\alpha}$ centroid position of MOS1,2 and PN data reveals that it is located at $1.8503 \pm 0.0012 \mathrm{keV}$ and $1.8298 \pm 0.0015 \mathrm{keV}$, respectively (the errors are $1 \sigma \chi^{2}$ ). For the $\mathrm{Fe}_{\alpha}$ line, we found $6.495 \pm 0.048 \mathrm{keV}$ and $6.442 \pm 0.025 \mathrm{keV}$, respectively. The discrepancies in the lines locations are about $1 \%$. Nevertheless, there are indications that the PN instrument has small gain problems and sometimes requires a shifting of the PN energy grid (private communications with Werner; Werner et al. 2006; de Plaa et al. 2004). In our study, we were unable to find a fitting model with sensible parameters of the emitting plasma for the PN data, while a spectral model for the MOS data provided reasonable and satisfactory plasma parameters.

The EPIC data was slightly affected by background flaring ("soft protons"). As a result, we cut out $3.4 \mathrm{ks}$ of the total observation. The MOS1 observation was completed in a small window mode, whereas the MOS2 data were acquired in full frame mode. In MOS1 we were therefore unable to select a region for extracting background spectra. For the MOS2, this was possible, but we found that the background correction was too small and that following its application the fitted parameters were unchanged. For that reason, we did not include background subtraction in our final analysis.

The RGS is a slitless spectrometer. For an extended source, this implies that the spectrum is smeared by the image of the source itself. For SMC/LMC remnants, the smearing is modest, but present, and it gives rise to a change in the line spread function. For our analysis, we incorporated this effect into the response matrix by convolving the standard (point source) response matrix with the emissivity profile of the SNR, as obtained from archival Chandra observations. This procedure was also applied to the RGS data of SN 1006 (Vink et al. 2003). As we show, however, in the case of 0509-67.5 the Doppler line broadening is much larger than the broadening caused by the spatial extent of the remnant.

Apart from adapting the RGS response matrix, all reduction for both MOS and RGS data was completed using the standard XMM-Newton software package SAS version 7.1.0.

\section{Spectral models}

The X-ray spectra of the SNR were fitted with a non-equilibrium ionization (NEI) model, which is a part of the SPEX fitting package (Kaastra et al. 1996). The package does not include a plane shock model (which takes into account temperature and ionization timescale gradients), but it contains the most complete and up-to-date set of atomic data. The use of a single ionization timescale NEI model is justified in some cases, when one needs to obtain approximate estimates of the basic SNR properties (e.g. Cassam-Chenaï et al. 2004). The emission measure $n_{\mathrm{H}} n_{\mathrm{e}} V$ in such a model can be used to estimate the circumstellar medium density. Since continuum emission originates mostly in the shocked CSM, the value of $n_{\mathrm{H}}$ can provide us with a direct estimate of the unshocked environment density (depending on the assumed equation of state).

Combined fitting of the spectra from the RGS and EPIC (MOS1 and MOS2) devices is presented in Fig. 2. 


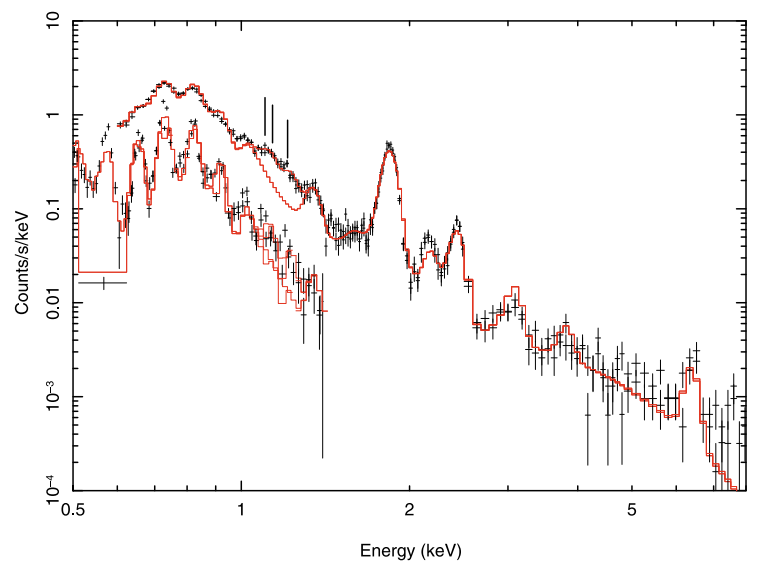

Fig. 2. MOS1, MOS2 and RGS data fitted with the NEI model, the Fe K line is fitted separately: an NEI component with $k T=3.5 \mathrm{keV}$ and $n_{\mathrm{e}} t=10^{9} \mathrm{~s} / \mathrm{cm}^{3}$. Vertical lines show the location of three Gaussians, which substitute the missing in SPEX model Fe L lines at the energies $1.102,1.125$, and $1.226 \mathrm{keV}$.

\subsection{EPIC data}

\subsubsection{Spectra parameters}

To adequately fit the spectra, we needed at least two thermal components: one NEI component is for the bulk of the X-ray line emission, and another is to fit the Fe-K emission around 6.5 keV (see Sect. 3.1.2 for details but this component). We also investigated a model with an additional power law continuum, since Warren \& Hughes (2004) reported indirect evidence for non-thermal continuum, probably synchrotron emission from $>\mathrm{TeV}$ electrons. The plasma parameters for the two models are indicated in Table 1 as (th) and (th+pow). In addition, we extracted the spectrum from the enhanced SW region of the remnant. In general, the parameters agree with those obtained by Warren \& Hughes (2004). The enhanced SW region shows higher ionization timescale, probably reflecting a higher density.

Using the value of the emission measure, we can estimate the density of the CSM. The hydrogen emission measure equals (Table 1, MOS+RGS) EM $=n_{\mathrm{e}} n_{\mathrm{H}} V=1.5 \times 10^{58} \mathrm{~cm}^{-3}$ and the remnant's size is $R=3.6 \mathrm{pc} \simeq 10^{19} \mathrm{~cm}$. Assuming that the emitting shell has a width of $\Delta R=R / 12$, so that the volume $V_{X}=$ $\pi R^{3} / 3 \simeq 10^{57} \mathrm{~cm}^{3}$, we obtain $n_{\mathrm{e}} n_{\mathrm{H}} \simeq 15 \mathrm{~cm}^{-6}$. Furthermore, by assuming that all matter is ionized $n_{\mathrm{H}} \simeq 4 \mathrm{~cm}^{-3}$, we measure the CSM number density for the remnant to be $n_{\mathrm{CSM}} \simeq 1 \mathrm{~cm}^{-3}$ for the neutral circumstellar environment, which appears to be the case for SNR 0509-67.5 (Ghavamian et al. 2007). Here, we have assumed that the shock compression ratio is 4 for non-relativistic matter with an adiabatic index $\gamma=5 / 3$. However, cosmic ray (CR) acceleration may play an important role in SNR dynamics. For a cosmic-ray-dominated shock, the adiabatic treatment of a relativistic gas $(\gamma=4 / 3)$ produces a compression factor of 7. Cosmic ray escape may even increase the compression ratio beyond 7 (Berezhko \& Ellison 1999). Tycho's SNR provides some observational evidence for high compression factors (Warren et al. 2005). In the case of 0509-67.5, the pre-shock density could therefore be as low as $n_{\mathrm{CSM}} \lesssim 0.6 \mathrm{~cm}^{-3}$.

Two additional sources of uncertainty concerning the preshock density are the presence or absence of a non-thermal continuum, and its chemical composition. Our above estimate assumes that most of the continuum is bremsstrahlung from hydrogen-electron collisions, whereas it is quite likely that parts

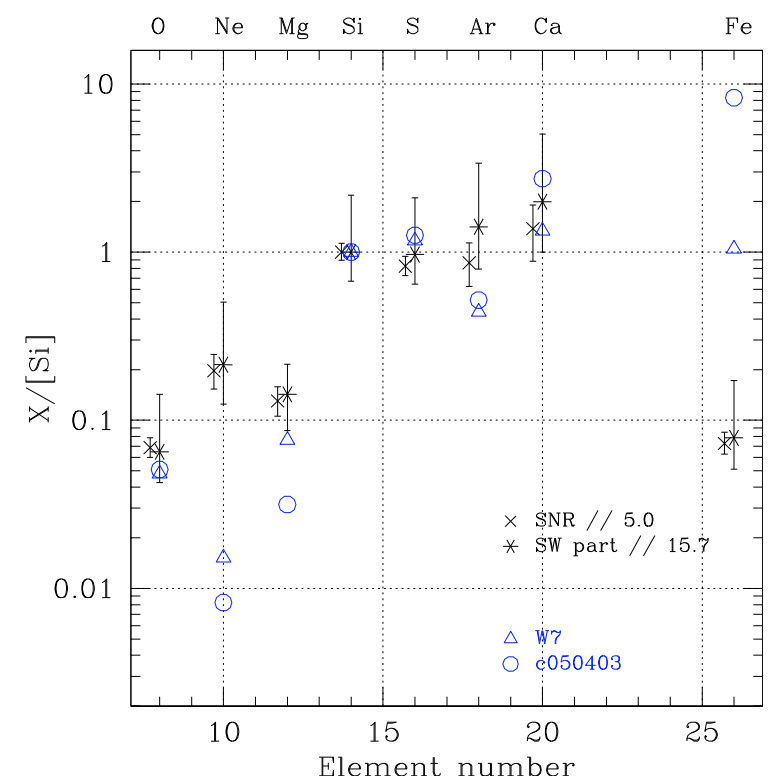

Fig. 3. Abundances of the entire remnant and its SW region, obtained by fitting of the EPIC (MOS1 and MOS2) spectrum. Abundances for the classical W7 (Iwamoto et al. 1999) and delayed-detonation model c050403m (Woosley et al. 2007) are also shown. Theoretical abundances are taken from numerical simulations (described in main text) that include swept-up $\left(\sim 0.7 M_{\odot}\right)$ ejecta and shocked LMC circumstellar medium. All abundances are with respect to solar abundances. Errors are $1 \sigma$ rms. The silicon abundance (with respect to solar) for the entire SNR is 5.0 and for the SW part of it is 15.7.

of the remnant consist of pure metal plasmas, in which case bremsstrahlung originates in metal ions and the electrons that they provide, which in general lowers the density estimate. Therefore, our estimate of $n_{\mathrm{CSM}} \simeq 1 \mathrm{~cm}^{-3}$ should be considered an upper limit.

Abundances of chemical elements for the entire SNR (thermal and nonthermal continuum) and for its enhanced SW part in the simple NEI model are presented in Fig. 3. The abundances of some theoretical explosion models (Iwamoto et al. 1999; Woosley et al. 2007) are also shown. The accounting for the nonthermal continuum does not create a considerable difference in the abundances set, but naturally it makes the remnant more metal-rich. One can see from the figure that the SW region is more metal abundant compared to overall remnant values (2-3 times higher amount of metals).

The SPEX fitting shows no evidence for interstellar absorption, which means that the value of $N_{\mathrm{H}}$ is low for this remnant. The column density value found by Warren \& Hughes (2004) is $N_{\mathrm{H}} \simeq(5-9) \times 10^{20} \mathrm{~cm}^{-2}$, which is indeed the lowest value measured for all known LMC supernovae remnants (Hughes et al. 1998). This could suggest that the remnant is located on the near side of the LMC.

The power law model for the continuum infers that the nonthermal flux equals $F_{1 \mathrm{keV}}^{X}=(2.3 \pm 0.3) \times 10^{-4} \mathrm{ph} / \mathrm{s} / \mathrm{cm}^{2} / \mathrm{keV}$ for a power index of $\Gamma=3.5 \pm 0.1$, which is steeper than expected for this type of remnant, e.g. Tycho SNR (cf. Tycho's SNR or Cas A, studied by Cassam-Chenaï et al. 2006; Vink \& Laming 2003). This value is close to that measured by Warren \& Hughes (2004), i.e. $3.25 \pm 0.18$. The extrapolation of the non-thermal radio flux (Hendrick \& Reynolds 2001) to X-ray range yields $F_{1 \mathrm{keV}}=1.4 \times 10^{-2} \mathrm{ph} / \mathrm{s} / \mathrm{cm}^{2} / \mathrm{keV}$. Since the X-ray synchrotron spectrum is likely to be affected by synchrotron losses, this flux should be considered as an upper limit. 
Table 1. Parameters for the SNR 0509-67.5 (last column contains data for the southwest region), EPIC (MOS1,2) and RGS data. Errors are 1 sigma $\left(\chi^{2}\right.$-distribution). th - basic (single ionization timescale) NEI model, th+pow - NEI with power law continuum.

\begin{tabular}{llllll}
\hline \hline Parameter & th, MOS+RGS & th, MOS & th+pow, MOS+RGS & th+pow, MOS & SW, th, MOS \\
\hline$n_{\mathrm{e}} n_{\mathrm{H}} V \times 10^{58}, \mathrm{~cm}^{-3}$ & $1.15_{-0.12}^{+0.12}$ & $0.98_{-0.10}^{+0.10}$ & $0.55_{-0.16}^{+0.15}$ & $0.95_{-0.10}^{+0.11}$ & $0.07_{-0.04}^{+0.05}$ \\
$k T, \mathrm{keV}$ & $4.01_{-0.18}^{+0.23}$ & $5.02_{-0.28}^{+0.33}$ & $4.55_{-0.20}^{+0.22}$ & $4.98_{-0.30}^{+0.30}$ & $4.59_{-0.45}^{+0.54}$ \\
$n_{\mathrm{e}} t \times 10^{10}, \mathrm{~s} / \mathrm{cm}^{3}$ & $1.41_{-0.03}^{+0.03}$ & $1.62_{-0.05}^{+0.05}$ & $1.63_{-0.04}^{+0.04}$ & $1.60_{-0.06}^{+0.05}$ & $1.52_{-0.08}^{+0.08}$ \\
$\sigma_{v}, \mathrm{~km} \mathrm{~s}^{-1}$ & $6030 \pm 170$ & $5700 \pm 220$ & $5050 \pm 180$ & $5350 \pm 240$ & $6100 \pm 500$ \\
$\chi^{2} /$ d.o.f. & 2.61 & 1.42 & 2.29 & 1.37 & 1.66 \\
\hline
\end{tabular}

\subsubsection{Fe K emission}

Despite the low amount of iron in the shocked ejecta obtained in a single ionization timescale NEI model, the spectrum has a pronounced $\mathrm{Fe} \mathrm{K}$ feature. Its centroid is located at $6.50 \pm 0.05 \mathrm{keV}$, which may correspond to ions from Fe VII (Kaastra \& Mewe 1993) with $n_{\mathrm{e}} t \simeq 10^{9} \mathrm{~s} / \mathrm{cm}^{3}$, to Fe XVII with $n_{\mathrm{e}} t \lesssim 10^{10} \mathrm{~s} / \mathrm{cm}^{3}$. We fitted the Fe $\mathrm{K}$ line separately with the temperature $k T=$ $3.5 \mathrm{keV}$ and ionization timescale $n_{\mathrm{e}} t=10^{9} \mathrm{~s} / \mathrm{cm}^{3}$. The ionization time is uncertain, but it is impossible to fit the Fe K emission using a component with $n_{\mathrm{e}} t>2 \times 10^{9} \mathrm{~s} / \mathrm{cm}^{3}$, since then the spectrum would have exhibited far more Fe L emission than observed. Given the low $n_{\mathrm{e}} t$, it is likely that the Fe $\mathrm{K}$ emission is associated with pure Fe, shocked recently by the reverse shock.

The separate fitting of the Fe $\mathrm{K}$ feature allows us to estimate the amount of iron layer of the supernova ejecta swept up by the reverse shock. Assuming that the pressure and temperature do not vary significantly within the shell, and the electron density $n_{\mathrm{e}}$ therefore remains approximately constant, we can employ the following relation $n_{\mathrm{Fe}} V_{\mathrm{Fe}} \sim\left(E M_{\mathrm{Fe}} / E M_{X}\right)\left(n_{\mathrm{H}} V_{\mathrm{X}}\right)$.

The emission measure value of the pure iron NEI component, $E M_{\mathrm{Fe}}=n_{\mathrm{e}} n_{\mathrm{Fe}} V_{\mathrm{Fe}} \simeq 4 \times 10^{54} \mathrm{~cm}^{-3}$, the basic NEI component, $E M_{\mathrm{X}}=n_{\mathrm{e}} n_{\mathrm{H}} V_{\mathrm{X}} \simeq 10^{57} \mathrm{~cm}^{-3}$, and also the density $n_{\mathrm{H}} \simeq 4 \mathrm{~cm}^{-3}$ and the volume $V_{\mathrm{X}} \simeq 10^{57} \mathrm{~cm}^{3}$ of the emitting shell, enable us to estimate the swept-up iron in the remnant of $M_{\mathrm{Fe}}=56 m_{U} n_{\mathrm{Fe}} V_{\mathrm{Fe}} \sim 0.05 M_{\odot}\left(\right.$ with $m_{U}=1.66 \times 10^{-24} \mathrm{~g}$, $\left.M_{\odot}=2 \times 10^{33} \mathrm{~g}\right)$. This is less than $10 \%$ of the amount of iron in a typical Ia SN model (Nomoto et al. 1984; Iwamoto et al. 1999).

\subsubsection{The line emission deficit around $1.2 \mathrm{keV}$}

The EPIC (MOS1,2) models do not fit an excess of emission around $1.2 \mathrm{keV}$. This feature is also present in the EPIC-pn spectra, and is visible weakly in the RGS spectra. Therefore this excess is unlikely to be caused by calibration errors or background features but is probably real. It is possible to fit this region of the spectrum, but only if we allow for unrealistic overabundance of either $\mathrm{Na}$ (approximately 5 times of $\mathrm{Si}$ ) or $\mathrm{Ni}$ (4 times of iron).

In our view, the possible cause of the excess is the uncertainty in the SPEX atomic database for the Fe-L emission. The Fe-L emission of SNR 0509-67.5 is dominated by Fe XVII. The excess therefore probably is due to incompleteness of the atomic database for Fe XVII, in particular due to line emission from the $n=4$ to $n=2$ level. This is corroborated by the fact that the series limit of Fe XVII is at $1.26 \mathrm{keV}$, which also roughly marks the end of the emission excess. Some $n=4 \rightarrow 2$ lines are present in the SPEX code, but their emission strengths may be underestimated. This may not be surprising, since the code has been tested primarily on collisional equilibrium sources, whereas in
NEI, $n=4 \rightarrow 2$ lines may be enhanced due to non-equilibrium effects, such as the inner shell ionization of Fe XVI ${ }^{1}$.

\subsection{RGS data}

The high resolution RGS spectra allowed us to evaluate the velocity broadening of the X-ray emission lines and estimate independently the CSM density. For the spectrum in the energy range $0.3-2.5 \mathrm{keV}$ we find $\sigma_{v}=4900 \pm 420 \mathrm{~km} \mathrm{~s}^{-1}$ (which is the preferred value since it is based on the RGS data only). This provides an upper limit to the age of the SNR of $t \lesssim R / v_{\text {shock }} \simeq$ $540 \pm 50 \mathrm{yr}$ (assuming $v_{\text {shock }}=4 / 3 \sigma_{v}$ ).

The first column in Table 1 shows the best-fit parameters for the joint MOS+RGS analysis. However, we also fitted the RGS spectra separately with a single NEI model. In this case, the fitted parameters were quite different, in particular the plasma temperature $k T_{\mathrm{e}}=0.75_{-0.09}^{+0.33} \mathrm{keV}$ and ionization timescale $n_{\mathrm{e}} t=$ $\left(1.28_{-0.33}^{+0.25}\right) \times 10^{10} \mathrm{~s} / \mathrm{cm}^{3}$; this is probably because the RGS is mostly sensitive to energies $0.5-1 \mathrm{keV}$, and therefore misses the contributions from lines that emit at higher temperatures and the continuum. The OVII lines around $0.55 \mathrm{keV} / 22 \AA$ for example, have far more statistical weight in the RGS spectra. The OVII line emission probably has contributions from a lower temperature plasma, which is not detected up by the MOS spectra. The difference in best-fit parameters points to the presence of temperatures gradients in the SNR.

Ideally, these gradients should be incorporated into a more complete model of the X-ray emission from SNR 0509-67.5. We attempted to derive such a model by fitting a joint MOSRGS model with 3 NEI components, one for the low temperature plasma, one for the bulk of the MOS spectrum, and one for the Fe-K line emission. However, it became difficult to find a unique, satisfying solution, given the complexity of the parameter space and the need for some arbitrary choices. For example, it is unclear how to treat the abundances; we assume one set of abundances for the first two NEI components, but then do not allow for possible abundance gradients, which are likely to be present. In short, we have reached the limit of the possible multiple components. The RGS data, however, demonstrate clearly that the models in Table 1 are incomplete.

An advantages of the high spectral resolution of the RGS data is that it is possible to obtain an estimate of the nitrogen abundance. Nitrogen is not an ejecta product of Type Ia supernovae, so any nitrogen must originate in the shocked CSM. With the emission measure, the nitrogen abundance therefore enables an alternative estimate of the pre-shock CSM density to be derived, avoiding some of the confusion caused by the mixture of pure metal, ejecta components.

1 We discussed this issue with Dr. Kaastra, one of the authors of the SPEX code, who agrees that this may explain the lack of a good fit around $1.2 \mathrm{keV}$. 

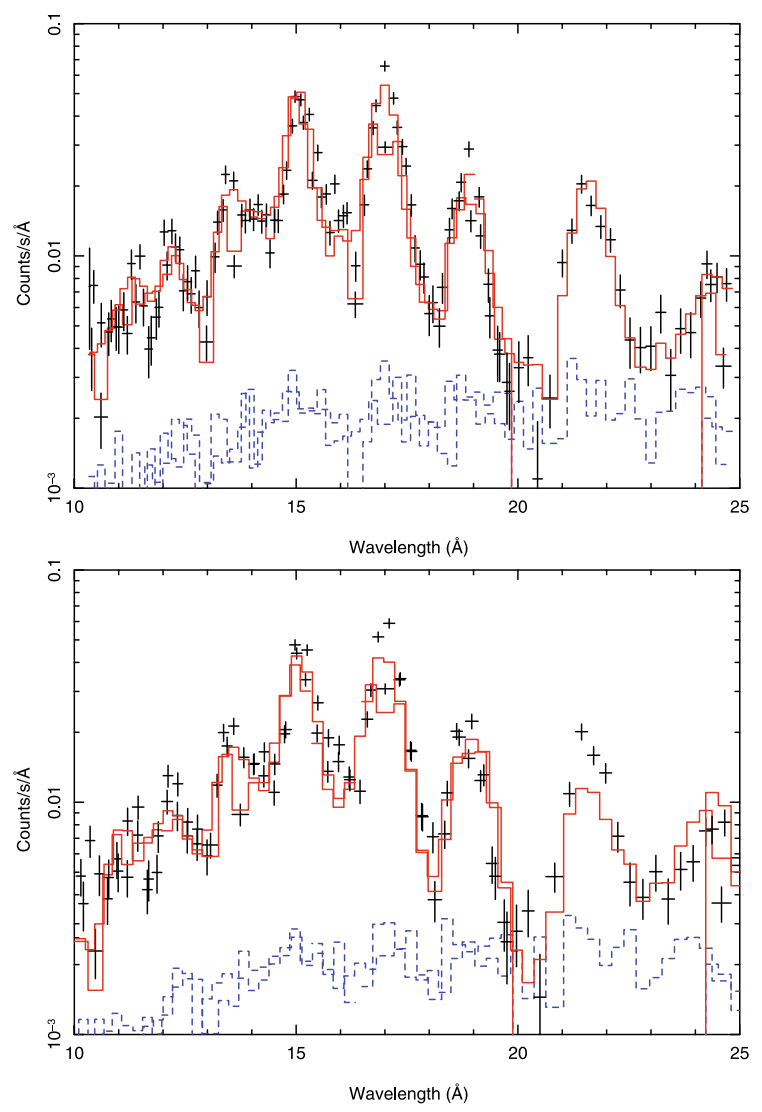

Fig. 4. Top: the RGS spectra with best-fit NEI model. Bottom: the RGS spectra and the MOS+RGS best-fit NEI model.

The hydrogen emission measure of the RGS fit of $n_{\mathrm{e}} n_{\mathrm{H}} V=$ $4 \times 10^{59} \mathrm{~cm}^{-3}$ and the emitting volume of $V_{X} \simeq 10^{57} \mathrm{~cm}^{3}$ correspond to $\left[n_{\mathrm{e}} n_{\mathrm{H}}\right]^{\mathrm{RGS}} \simeq 400 \mathrm{~cm}^{-6}$. Taking into account the bestfit RGS amount of nitrogen $X_{N}^{\mathrm{RGS}}=0.01$ and the typical LMC abundance $X_{N}^{\mathrm{CSM}}=0.4$ (both in solar units), for the circumstellar matter we derive $\left[n_{\mathrm{e}} n_{\mathrm{H}}\right]^{\mathrm{CSM}}=\left[n_{\mathrm{e}} n_{\mathrm{H}}\right]^{\mathrm{RGS}}\left(X_{N}^{\mathrm{RGS}} / X_{N}^{\mathrm{CSM}}\right)=$ $10 \mathrm{~cm}^{-6}$. For the shocked CSM (LMC abundances), we therefore measure $n_{\mathrm{H}}=3 \mathrm{~cm}^{-3}$, which corresponds to $n_{\mathrm{CSM}}=$ $0.4-0.8 \mathrm{~cm}^{-3}$.

\section{Numerical models}

To simulate the remnant evolution and compare with observed data, we employed the hydrodynamical code SUPREMNA, which is described in detail by Sorokina et al. (2004). The code assumes spherical symmetry, but incorporates many relevant physical processes; without these processes it would indeed be impossible to predict accurately peculiarities of the SNR radiation, such as the time-dependent ionization, inner-shell collisional ionization, possible difference in temperatures of electrons and ions, the influence of radiative losses, the account of electron thermal conduction and nonthermal particles. The calculation of line emission was based on the methods of Gaetz \& Salpeter (1983) and Mewe et al. (1985). The introduction of inner-shell ionization processes is described by Kosenko (2006).

To reproduce the observed remnant in numerical simulations we considered various physical conditions of the CSM and different explosion models, for example deflagration W7 (Nomoto et al. 1984) and some of the "mildly-mixed" delayed-detonation models (Woosley et al. 2007).
Following the estimates of the CSM density derived from the SPEX spectral fitting, we surround the remnant with uniform medium of $\rho_{\mathrm{CSM}}=3 \times 10^{-25} \mathrm{~g} / \mathrm{cm}^{3}$. In this set up we found that at the age of $t \sim 400$ years the modeled remnant's radius reaches $R \sim 3.6 \mathrm{pc}$, and the typical velocity of the plasma is $v \sim 5000 \mathrm{~km} \mathrm{~s}^{-1}$. These parameters agree with the observational measurements for the SNR 0509-67.5. Model temperatures and $\mathrm{X}$-ray spectra are discussed in detail below.

\subsection{Basic modeling}

First, we used a "basic" approach, as described by Sorokina et al. (2004) where electrons and ions are heated partially due to artificial viscosity and the degree of electrons heating is controlled by a parameter $q_{i}$, such that $P_{\mathrm{i}}=P_{\mathrm{i}}$ (thermal) $+q_{\mathrm{i}} Q$ and $P_{\mathrm{e}}=P_{\mathrm{e}}$ (thermal) $+\left(1-q_{\mathrm{i}}\right) Q$, where $P_{\mathrm{e}}, P_{\mathrm{i}}$ are the electrons and ions pressure respectively, $Q$ is the artificial viscosity, for the case when only collisional energy exchange occurs, and $q_{\mathrm{i}}=1-m_{\mathrm{e}} / m_{\mathrm{i}}$, where $m_{\mathrm{e}}$, and $m_{\mathrm{i}}$ are the electron and ion masses, respectively. We note that since $q_{\mathrm{i}}$ controls the efficiency of electron heating (their temperature can vary theoretically between $10^{-4} T_{\mathrm{i}}$ and $T_{\mathrm{i}}$ ), this parameter has a strong influence on the X-ray spectrum behavior, i.e. the line fluxes with respect to the free-free continuum.

In this framework, we found that the best-fit function (in terms of $\chi^{2} /$ d.o.f.) for the observed spectrum provides the value of $q_{\mathrm{i}}=0.99$, which yields the typical ratio of $T_{\mathrm{e}} / T_{\mathrm{i}} \simeq 3 \times 10^{-3}$ for the case of W7 explosion model. We also considered a library of thermonuclear explosion models by Woosley et al. (2007). We employed several of these models with various amounts of iron and intermediate mass elements (IME). We found that the explosion of a "mildly-mixed" delayed detonation model with $E=1.4 \times 10^{51} \mathrm{ergs}, M_{\mathrm{Ni}}=0.5 M_{\odot}, M_{\mathrm{Fe}}=0.4 M_{\odot}$, and $M_{\mathrm{IME}}=0.3 M_{\odot}(\mathrm{c} 050403 \mathrm{~m}$ model in Woosley et al. 2007 , notation) provides the best-fit function parameters for the observed EPIC MOS spectrum of the SNR with $q_{\mathrm{i}}=0.9$ and therefore that $T_{\mathrm{e}} / T_{\mathrm{i}} \simeq 3 \times 10^{-2}$. These values appear to be in reasonable agreement with the relation $T_{\mathrm{e}} / T_{\mathrm{i}} \propto v_{\mathrm{S}}^{-2}$, where $v_{\mathrm{S}}$ is the shock velocity, proposed by Ghavamian et al. (2000). Assuming the SNR 0509-67.5 shock front speed of $v_{\mathrm{S}} \simeq 4 / 3 v \simeq 6000 \mathrm{~km} \mathrm{~s}^{-1}$, we should expect $T_{\mathrm{e}} / T_{\mathrm{i}}=6 \times 10^{-3}$ according to Ghavamian et al. (2000, Fig. 2).

The simulated (for these two explosion models) and the observed X-ray spectra are presented in Fig. 6. We note that all of our models exhibit a noticeable lack of flux in the region of Fe L complex in comparison to the observed spectrum. This is caused by some incompleteness in the atomic data set for Fe L emission employed in our package, as verified by comparing directly a single NEI model as obtained by the SUPREMNA code and SPEX. This issue will be addressed in future updates of the SUPREMNA code.

At higher densities in the CSM, there is no Fe L emission deficit, but in these models the expansion rate of the shell and the radius of the contact discontinuity disagree with the observed values of the line broadening and the remnant size. The value of CSM density was therefore chosen to satisfy the observed velocity of the emitting ejecta, the age, and the geometrical size (assuming that the observed edge of the remnant corresponds to the location of the contact discontinuity) of the remnant for the considered explosion mechanisms. Figure 5 shows the maximum velocity of the shocked ejecta and the position of the contact discontinuity depending on the assumed value of CSM density for the delayed detonation explosion model c050403 at the age of 350 (left panels) and 400 (right panels) years. The 


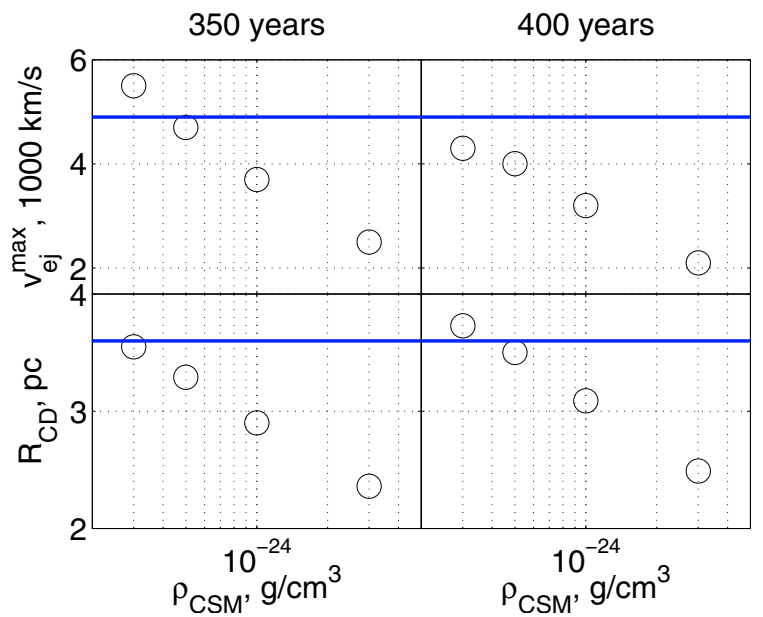

Fig. 5. Maximum velocity of the shocked ejecta ( $v_{\mathrm{ej}}^{\max }$, upper row) and position of the contact discontinuity ( $R_{\mathrm{CD}}$, lower row) depending on the value of CSM density for 350 (left column) and 400 years (right column). The explosion model is the delayed detonation model c050403 (Woosley et al. 2007) with $E=1.4 \times 10^{51} \mathrm{erg}$. The observed values of the velocity and radius of the remnant are also outlined.

figure indicates that for an assumed age of 400 years, the CSM density of $3 \times 10^{-25} \mathrm{~g} / \mathrm{cm}^{3}$ provides a reasonable compromise between velocity and radius. An assumption that the remnant may be as young as 350 years, allows the CSM to be denser than $5 \times 10^{-25} \mathrm{~g} / \mathrm{cm}^{3}$, but in this case the synthetic X-ray spectra disagree with the observed ones (there are major discrepancies in Fe L emission and Si K and S K lines fluxes). A more energetic $\left(E>1.4 \times 10^{51} \mathrm{ergs}\right)$ progenitor could shift the allowable values of the density to higher ranges. We checked several of these models, which contain more ${ }^{56} \mathrm{Ni}$ and could be consistent with the optical spectrum of the supernova, as extracted from the light echo (Rest et al. 2008). However, these models contain too little $\mathrm{Si}$ and $\mathrm{S}$ to fit their emission lines.

X-ray spectra of the simulated and observed (merged MOS1 and MOS2, crosses) remnants are presented in Fig. 6. The theoretical spectra are based on $\mathrm{W} 7\left(T_{\mathrm{e}} / T_{\mathrm{i}} \simeq 0.003, \chi^{2} /\right.$ d.o.f. $\simeq 27$, dashed line) and delayed-detonation c050403m $\left(T_{\mathrm{e}} / T_{\mathrm{i}} \simeq 0.03\right.$, $\chi^{2} /$ d.o.f. $\simeq 19$, solid line) explosion models with $\rho_{\mathrm{CSM}}=$ $3 \times 10^{-25} \mathrm{~g} / \mathrm{cm}^{3}$, and age $t=400$ years.

Table 2 lists some basic properties of these models. Some of the numerical values in the table are upper limits or are just typical for the corresponding cases. Errors are presented where appropriate.

\subsection{Accounting for the cosmic rays}

We introduced an additional component representing the pressure and energy from (relativistic) cosmic ray particles, in a way that could be implemented easily within the current framework of this code. This was achieved by altering (softening) the equation of state as follows:

$$
\left\{\begin{array}{l}
P=P_{\mathrm{g}}+\frac{1}{3} \hat{a} T^{4} \\
E=E_{\mathrm{g}}+\hat{a} T^{4}
\end{array}\right.
$$

where $P_{\mathrm{g}}$ is the gas pressure, $E_{\mathrm{g}}$ is the gas internal energy, $T$ is the "temperature", and $\hat{a}$ is a free parameter mimicking the contribution of the relativistic particles. Effectively this means, that some part of the energy is transferred into relativistic particles, which behave in a similar way to a photon gas.

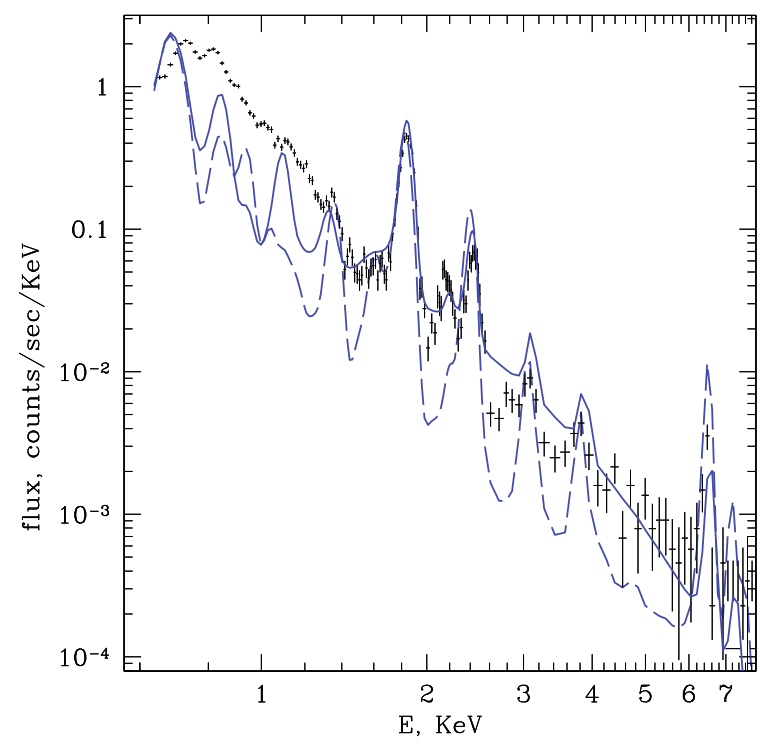

Fig. 6. X-ray spectra of the simulated and the observed (merged MOS1 and MOS2, crosses) remnants. The theoretical spectra are based on W7 $\left(T_{\mathrm{e}} / T_{\mathrm{i}} \simeq 0.003, \chi^{2} /\right.$ d.o.f. $\simeq 27$, dashed line $)$ and delayed-detonation c050403m $\left(T_{\mathrm{e}} / T_{\mathrm{i}} \simeq 0.03, \chi^{2} /\right.$ d.o.f. $\simeq 19$, solid line $)$ explosion models. $\rho_{\mathrm{CSM}}=3 \times 10^{-25} \mathrm{~g} / \mathrm{cm}^{3}$, age $t=400$ years.

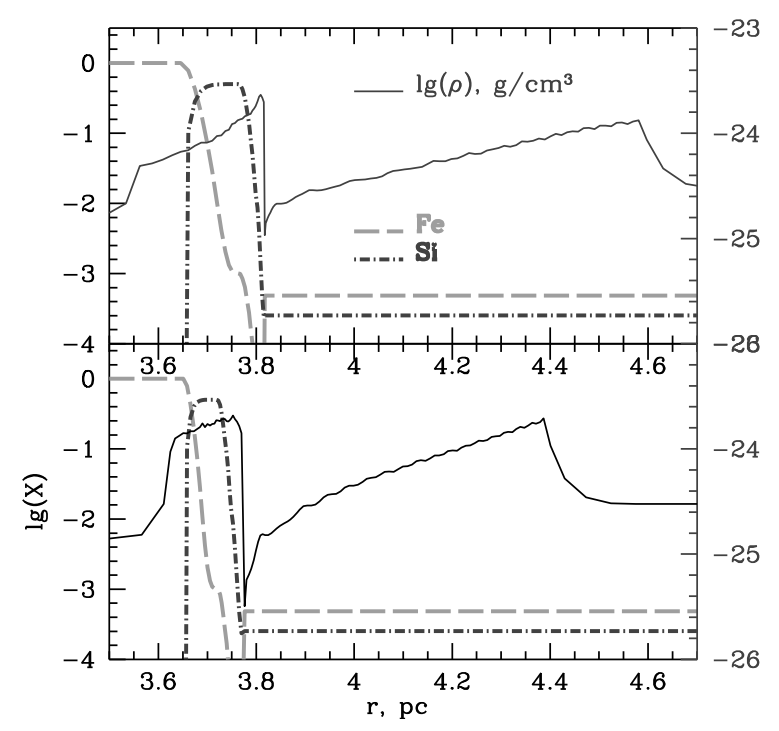

Fig. 7. Density profiles (solid lines) against radius for the hydrodynamical models of age 400 years and for the delayed detonation explosion mechanism c050403m. Upper panel: basic set up, lower pane: the set up with the relativistic correction in the equation of state (Eq. (1)). Also the distributions of $\mathrm{Si}$ (black dash-dotted line) and $\mathrm{Fe}$ (gray dashed line) in the ejecta are shown.

Density profiles for the cases with and without this relativistic correction for the delayed-detonation c050403m (Woosley et al. 2007) model are presented in Fig. 7. We note that in the case of the relativistic alteration of the equation of state, the density jump at the forward shock is enhanced, compared with the basic set-up, and the density profile of the ejecta behind the reverse shock also becomes affected.

In reality the cosmic ray contribution to the internal energy however may be different for the forward and reverse shock. In the case of the SNR Cas A, Helder \& Vink (2008) established that the reverse shock can accelerate cosmic ray particles up to energies of a few $\mathrm{TeV}$. 
Table 2. Observed properties of the SNR 0509-67.5 and the properties of the numerical models: the one with the basic set up and one with a relativistic component (Eq. (1)). The table columns are: $R$ - visible remnant's radius (in the models this is the position of the contact discontinuity), $\mathrm{t}$ - the age, $n_{\mathrm{CSM}}$ - circumstellar number density, $v$ - velocity of the shocked ejecta (maximum value for the numerical models), $k T_{\mathrm{e}}-\mathrm{electron}$ temperature (for numerical models it is the maximum temperature), $k T_{\mathrm{i}}-$ maximum ion temperature, $M_{\mathrm{Fe}}-$ an estimate of the swept up iron mass (in solar masses), $N_{\mathrm{H}}$ - column density on the line of sight (derived from XSPEC, Arnaud 1996, spectral fitting).

\begin{tabular}{lllllllll}
\hline \hline Parameters & $R, \mathrm{pc}$ & $t, \mathrm{yrs}$ & $n_{\mathrm{CSM}} \mathrm{cm}^{-3}$ & $v, \mathrm{~km} \mathrm{~s}^{-1}$ & $k T_{\mathrm{e}}, \mathrm{keV}$ & $k T_{\mathrm{i}}, \mathrm{keV}$ & $M_{\mathrm{Fe}}, M_{\odot}$ & $N_{\mathrm{H}} 10^{21} \mathrm{~cm}^{-2}$ \\
\hline SNR 0509-67.5 & 3.6 & $\lesssim 500$ & $0.4-0.6$ & $4900 \pm 400$ & $2.5-3.6$ & - & $\sim 0.05$ & - \\
W7(basic) & 3.6 & 400 & 0.1 & $\lesssim 4600$ & $0.5-3.6$ & $150-2500$ & $\sim 0.12$ & $0.7 \pm 0.3$ \\
c050403m(basic) & 3.7 & 400 & 0.1 & $\lesssim 4200$ & $1-1.5$ & $30-40$ & $\sim 0.36$ & $5.6 \pm 0.3$ \\
\hline W7(CR) & 3.6 & 400 & 0.1 & $\lesssim 4300$ & $1.8-1.9$ & $20-36$ & $\sim 0.12$ & $2.1 \pm 0.3$ \\
c050403m(CR) & 3.8 & 400 & 0.1 & $\lesssim 4700$ & $2-45$ & $30-300$ & $\sim 0.36$ & $2.6 \pm 0.3$ \\
\hline
\end{tabular}

In this set-up, a configuration with the cosmic-ray energy density $E_{\mathrm{CR}}=\hat{a} T^{4}$ of approximately $0.4 E_{\mathrm{g}}$ produce a model that also provides an accurate fit of the observed spectrum. The modeled and observed X-ray spectra for this approach are presented in Fig. 8.

Chevalier (1983) considered self-similar solutions for a mixture of a fluid with $\gamma=5 / 3$ and a relativistic gas (i.e., cosmic rays) with $\gamma=4 / 3$. The effective adiabatic index was determined by a constant fraction of cosmic rays in total pressure. Blondin \& Ellison (2001) used a constant value of effective $\gamma$ that occasionally assume to be even lower than $4 / 3$ to allow for a stronger compression ratio, which mimicked radiative shock waves. We note that our parametrization is simple in comparison with a true cosmic rays model and far richer than used by Chevalier (1983), and Blondin \& Ellison (2001) in their purely hydrodynamic studies. We do not assume a constant fraction of cosmic rays or a constant value of effective $\gamma$. Thermal conduction and radiative losses are fully included in a hydrodynamical scheme, in contrast with the vast majority of modern work on the subject. However, the validity of our simplified model of cosmic ray contribution requires further tests and a comparison with real supernova remnants and more sophisticated schemes modeling the remnants.

The value of the energy density found for relativistic particles $E_{\mathrm{CR}}$ can be used to estimate the intensity of the magnetic field in the remnant. By assuming that $E_{\mathrm{CR}}$ and $B^{2} / 8 \pi$ should be of the same order of magnitude, we derive $B=500 \pm 200 \mu G$. This value appears to be high for this SNR, but still within reasonable limits. For example, using the correlation $\rho_{\mathrm{CSM}} v_{S}^{3} \propto B^{2}$ from Vink (2006, Fig. 6), one might expect that $B \lesssim 100 \mu G$.

The typical properties of these models are also presented in Table 2. The difference between electron and ion temperatures is not significant: $T_{\mathrm{e}} / T_{\mathrm{i}} \simeq 0.03$, which still agrees with Ghavamian et al. (2000), where at $\gamma=4 / 3$ (relativistic equation of state) the SNR plasma velocities of approximately $4500 \mathrm{~km} \mathrm{~s}^{-1}$ correspond to $T_{\mathrm{e}} / T_{\mathrm{i}} \simeq 10^{-2}$.

\section{Discussions and concluding remarks}

The analysis of the XMM-Newton X-ray spectrum of the SNR 0509-67.5 has allowed us to estimate some basic parameters of the object and has revealed several peculiar characteristics.

First of all, we obtained reasonable and consistent estimates for the CSM density, using various methods: the EPIC MOS data fitting provided an estimates of the circumstellar medium density of $n_{\mathrm{CSM}} \lesssim 0.6 \mathrm{~cm}^{-3}$, and the RGS spectral fitting of the nitrogen abundance, which is not a product of thermonuclear explosion, measured $n_{\mathrm{CSM}}=0.4-0.8 \mathrm{~cm}^{-3}$. In the hydrodynamical simulations, we found a similar, but lower value of $n_{\mathrm{CSM}}=0.1 \mathrm{~cm}^{-3}$.

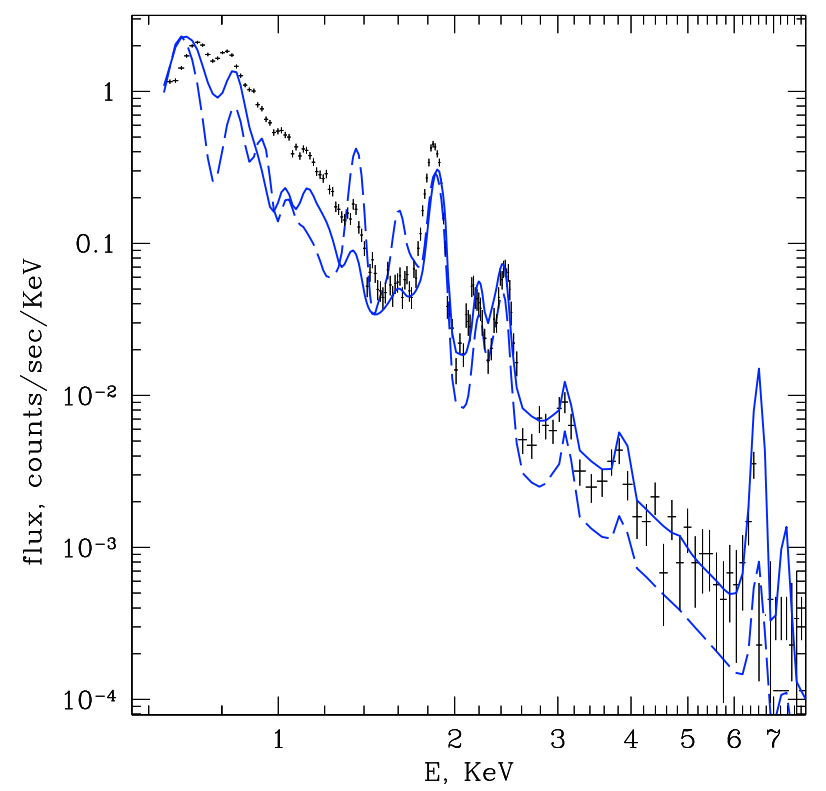

Fig. 8. X-ray spectra of the simulated and observed (merged MOS1 and MOS1) remnants. The theoretical spectra are based on the W7 $\left(\chi^{2} /\right.$ d.o.f. $\simeq 21$, dashed line $)$ and delayed-detonation $c 050403 \mathrm{~m}$ $\left(\chi^{2} /\right.$ d.o.f. $\simeq 13$, solid line $)$ explosion models, where $\rho_{\mathrm{CSM}}=3 \times$ $10^{-25} \mathrm{~g} / \mathrm{cm}^{3}$, age $t=400$ years, and $E_{\mathrm{CR}} / E_{\mathrm{g}} \simeq 0.4$.

The abundances deduced using a single ionization timescale NEI (Fig. 3) indicate that a swept-up amount of iron in the remnant is low. Moreover, the model was unable to reproduce the observed Fe K line, so the line was fitted separately. The centroid of the Fe K line was located at $6.50 \pm 0.05 \mathrm{keV}$, so it should be produced by low ionized ( $\leqslant$ XVII) ions of iron. This could mean that the reverse shock of the remnant recently reached and heated up the iron core of the supernova. The Fe $\mathrm{K}$ feature enables us to approximate the amount of heated iron to be $M_{\mathrm{Fe}} \sim 0.1 M_{\odot}$.

In the framework of a NEI model with a fixed singleionization timescale, the abundances of species lighter than aluminum tend to be overestimated, while the amount of heavy species (iron group) tends to be underestimated (Hughes et al. 1998). In the $\mathrm{W} 7$ model, for example, the $\mathrm{Mg} / \mathrm{Si}$ line flux ratio is higher than observed (Fig. 6, Fig. 8), while Fig. 3 demonstrates that the $\mathrm{Mg} / \mathrm{Si}$ abundance ratio is lower than the value measure from observations. The same is true for the iron abundance: Badenes et al. (2008), and Rest et al. (2008) showed that the remnant has a significantly high amount of iron, while the abundance of Fe, deduced from this simple fitting, is considerably low. We note, however, that most of the Fe has probably not yet been heated by the reverse shock. 
Using only abundances derived from a single-ionization timescale NEI model, it is difficult to determine the precise explosion mechanism. If we consider only species from $\mathrm{Si}$ to Ar, then a delayed-detonation model with $M_{\mathrm{Fe}}=0.9 M_{\odot}$ and an explosion energy of $1.4 \times 10^{51} \mathrm{erg}$ (Woosley et al. 2007) appears to be more appropriate (Fig. 3). A similar result is found by the comparison of observed spectra with spectra predicted by the numerical simulations completed in this study, and is in agreement with the results of Badenes et al. (2008), and Rest et al. (2008).

A separate study of the remnant's bright southwest region indicates that this area appears to be more metal-rich: our fitting shows that it is at least a factor of two more abundant in metals than the average metallicity measured for the remnant. This implies that either an asymmetric SNR explosion has occurred or an asymmetric CSM is present. We note that Ghavamian et al. (2007) found that this region has a blueshifted velocity excess, which provides support for an asymmetric explosion.

The numerical models agree with the overall properties of SNR 0509-67.5 inferred from the studies presented in Sect. 3, which are: the CSM density $\rho_{\mathrm{CSM}} \simeq 3 \times 10^{-25} \mathrm{~g} / \mathrm{cm}^{3}$, the age $t=350-400$ years, and the plasma velocities $v \simeq$ $4000-5000 \mathrm{~km} \mathrm{~s}^{-1}$. The ratio of electron-to-ion temperatures of $T_{\mathrm{e}} / T_{\mathrm{i}} \simeq 0.01$ agrees satisfactory with the expansion velocity of the remnant (Ghavamian et al. 2000), but depends heavily on the assumed explosion model (see also Badenes et al. 2006). The ejecta abundances determine electron population $n_{\mathrm{e}}$ and thus, the $\mathrm{X}$-ray spectral shape. The X-ray spectral shape is used to find the "best-fit" value of $T_{\mathrm{e}} / T_{\mathrm{i}}$.

The low value of $T_{\mathrm{e}} / T_{\mathrm{i}}$ and almost undetectable powerlaw emission highlight the apparently ineffective role of plasma instabilities and/or magnetic field at this stage in this object. Nevertheless, the simulations indicate that the energy of the relativistic particles is probably not negligible and may contribute up to $\sim 40 \%$ of thermal energy; the magnetic field (provided that $E_{\mathrm{CR}} \sim B^{2} / 8 \pi$ ) might therefore be slightly higher, approximately equal to $500 \mu G$, than the expected value of $\sim 100 \mu G$ based on the relation reported by Vink (2006), but within reasonable limits.

We developed satisfactory hydrodynamical models for the remnant, but many problems remain.

In our synthetic spectra some of the line centroids and line fluxes ratios did not fit the observations perfectly. This is due in part to the limited set of explosion models available and the appreciable number of free parameters (electron temperature, thermal conduction, magnetic fields, cosmic-ray particles, and other non-thermal and relativistic effects). For instance, a more energetic explosion model $\left(E>1.4 \times 10^{51} \mathrm{ergs}\right)$ could produce a supernova remnant of the age 350-400 years with the required high ejecta velocity, observed radius, and a more acceptable high value of CSM density (up to $10^{-24} \mathrm{~g} / \mathrm{cm}^{3}$ ). Such a model would however have to consist of a significant amount of ${ }^{56} \mathrm{Ni}$ and a small amount of $\mathrm{Si}$ and $\mathrm{S}$. The X-ray spectra produced by such an explosion model disagreed with the observed spectrum, which contains pronounced Si K and S K lines.

One of the shortcomings of the code is that for both the calculation of the X-ray emission and the reproduction of the observed spectrum about $1 \mathrm{keV}$, we need to update the atomic data in the Fe L code, which we plan to achieve in the near future.

To create reliable model of supernova remnants, further developments of the physics in hydrodynamical simulations are required. Self-consistent treatment of cosmic-ray acceleration is necessary. 3D simulations may shed light on the influence of various instabilities on the SNR dynamics. Further analysis of high resolution X-ray spectra is needed to evaluate the conditions inside other SNRs as well.

Acknowledgements. We are grateful to the referee C. Badenes for valuable comments which helped to improve the paper. We also thank P. Lundqvist for providing his X-ray code which is incorporated in the package SUPREMNA, J. Kaastra for helpful advice on X-ray line emission issues, M. Gilfanov for valuable pointers to X-ray data sources and S. Woosley for his set of SNIa models used in our work.

DK and JV are supported by a Vidi grant from the Netherlands Organization for Scientific Research (PI J. Vink), The work of DK is also partially supported in Russia by RFBR under grants 05-02-17480, 06-02-16025, 07-02-00961, and by Russian Leading Scientific School Foundation under grant RLSS-2977.2008.2.

SB is supported in Russia partly by grants RFBR 07-02-00830-a, RLSS3884.2008 .2 and by grant IB7320-110996/1 of the Swiss National Science Foundation.

\section{References}

Arnaud, K. 1996, in Astronomical Data Analysis Software and Systems V, ed. Jacoby G. and Barnes J., ASP Conf. Ser., 101, 17

Badenes, C., Borkowski, K., Hughes, J., Hwang, U., \& Bravo, E. 2006, ApJ, 645,1373

Badenes, C., Hughes, J. P., Bravo, E., \& Langer, N. 2007, ApJ, 662, 472

Badenes, C., Hughes, J. P., Cassam-Chenai, G., \& Bravo, E. 2008,

[arXiv:0801.4761]

Behar, E., Rasmussen, A. P., Griffiths, R. G., et al. 2001, A\&A, 365, 242

Berezhko, E., \& Ellison, D. C. 1999, ApJ, 526, 385

Blondin, J. M., \& Ellison, D. C. 2001, ApJ, 560, 244

Cassam-Chenaï, G., Decourchelle, A., Ballet, J., et al. 2004, A\&A, 414, 545

Cassam-Chenaï, G., Hughes, J. P., Ballet, J., \& Decourchelle, A. 2006, ApJ, 665, 315

Chevalier, R. A. 1983, ApJ, 272, 765

de Plaa, J., Kaastra, J. S., Tamura, T., et al. 2004, A\&A, 423, 49

den Herder, J. W., Brinkman, A. C., Kahn, S. M., Branduardi-Raymont, G., \& Thomsen, K. et al. 2001, A\&A, 365, 7

Gaetz, T. J., \& Salpeter, E. E. 1983, ApJS, 52, 155

Ghavamian, P., Blair, W. P., Sankrit, R., Raymond, J. C., \& Hughes, J. P. 2007, ApJ, 664, 304

Ghavamian, P., Laming, J. M., \& Rakowski, C. E. 2000, ApJ, 654, 69

Helder, E., \& Vink, J. 2008, ApJ, submitted

Hendrick, S. P., \& Reynolds, S. 2001, ApJ, 559, 903

Hughes, J. P., Hayashi, I., \& Koyama, K. 1998, ApJ, 505, 732

Iwamoto, K., Brachwitz, F., Nomoto, K., et al. 1999, ApJS, 125, 439

Kaastra, J., \& Mewe, R. 1993, ApJS, 97, 443

Kaastra, J., Mewe, R., \& Nieuwenhuijzen, H. 1996, in UV and X-ray spectroscopy of astrophysical and laboratory plasmas (K. Yamashita and T. Watanabe), 411

Kosenko, D. 2006, MNRAS, 369, 1407

Mewe, R., Gronenschild, E. H. B. M., \& van den Oord, G. H. J. 1985, A\&AS, 62, 197

Nomoto, K., Thielemann, F.-K., \& Yokoi, K. 1984, ApJ, 286, 644

Rasmussen, A. P., Behar, E., Kahn, S. M., den Herder, J. W., \& van der Heyden, K. 2001, A\&A, 365, 231

Rest, A., Suntzeff, N. B., Olsem, K., et al, 2005, Nature, 438, 1132

Rest, A., Matheson, T., Blondin, S., et al. 2008, [arXiv:0801.4762]

Sasaki, M., Gaetz, T. J., Blair, W. P., et al. 2006, ApJ, 642, 260

Sorokina, E., Blinnikov, S., Kosenko, D., \& Lundqvist, P. 2004, Astron. Lett., 30,737

Strüder, L., Briel, U., Dennerl, K., Hartmann, R., \& Kendziorra, E. et al. 2001, A\&A, 365, 18

Turner, M. J. L., Abbey, A., Arnaud, M., Balasini, M., \& Barbera, M. et al. 2001, A\&A, 365, 27

van der Heyden, K. J., Paerels, F., Cottam, J., Kaastra, J. S., \& BranduardiRaymont, G. 2001, A\&A, 365, 254

van der Heyden, K. J., Behar, E., Vink, J., et al. 2002, A\&A, 392, 955

van der Heyden, K. J., Bleeker, J. A. M., \& Kaastra, J. S. 2004, A\&A, 421, 1031

Vink, J. 2006, in The X-ray Universe 2005 (San Lorenzo de El Escorial, Madrid, Spain) ESA SP-604, [arXiv: astro-ph/0601131]

Vink, J., \& Laming, J. M. 2003, ApJ, 758, 584

Vink, J., Laming, J. M., Gu, M. F., Rasmussen, A., \& Kaastra, J. S. 2003, ApJ, 31,587

Warren, J. S., \& Hughes, J. P. 2004, ApJ, 608, 261

Warren, J. S., Hughes, J. P., Badenes, C., et al. 2005, ApJ, 634, 376

Werner, N., de Plaa, J., Kaastra, J. S., et al. 2006, A\&A, 449, 475

Woosley, S. E., Kasen, D., Blinnikov, S., \& Sorokina, E. 2007, ApJ, 662, 487 